\title{
The role of M-health applications in the fight against Alzheimer's: current and future directions
}

\author{
Abdelrahman Osman Elfaki, Mohammed Alotaibi \\ College of Computers and Information Technology, University of Tabuk, Tabuk, Saudi Arabia \\ Contributions: (I) Conception and design: AO Elfaki; (II) Administrative support: All authors; (III) Provision of study materials: All authors; (IV) \\ Collection and assembly of data: All authors; (V) Data analysis and interpretation: All authors; (VI) Manuscript writing: All authors; (VII) Final \\ approval of manuscript: All authors. \\ Correspondence to: Abdelrahman Osman Elfaki. College of Computers and Information Technology, University of Tabuk, P. O. Box 741, Tabuk, \\ Saudi Arabia. Email: abdelrahmanelfaki@gmail.com.
}

Background: Alzheimer's disease has become a very significant problem in the current era that is
very likely to worsen due to increasing life expectancy. The enormous potential of smart mobile devices
has attracted researchers and medical practitioners alike to develop and provide M-health solutions for
Alzheimer's. In this paper, we explore, analyze and investigate the works that have attempted to develop
M-health applications for Alzheimer's over the last 10 years in order to highlight current and future
directions, which it is hoped will assist medical practitioners and researchers to provide more solutions for
fighting Alzheimer's.

Methods: A systematic review approach was used in this study. Google Scholar is considered for searching the published papers for academic mobile applications and Google PlayStore is considered for non-academic mobile applications. More than 900 titles of various papers were reviewed. The selection of the papers was dictated by a set of inclusion and exclusion criteria.

Results: Current directions are identified by presenting the services and functions that are provided by M-health applications for Alzheimer's patients and the technologies that are used to do so. Current services are classified into four groups and the future directions for each of these groups are presented.

Conclusions: Numerous M-health applications that fight against Alzheimer's have been surveyed and analyzed by using scientific methodology in which inclusion and exclusion criteria have been used. Based on this analysis, we were able to present the current research foci and define potential future research directions.

Keywords: Mobile health applications; mobile technologies; Alzheimer's

Received: 13 May 2018; Accepted: 08 June 2018; Published: 08 August 2018.

doi: 10.21037/mhealth.2018.07.03

View this article at: http://dx.doi.org/10.21037/mhealth.2018.07.03

\section{Introduction}

Recently, mobile health or M-health has been receiving a lot of attention worldwide from patients, healthcare professionals, application developers, network service providers and researchers (1-4). Mobile phone software applications or apps are available for a variety of useful healthcare tasks such as symptom assessment, psychoeducation, resource location, and tracking of treatment progress (5). Ventola in (6) has outlined the uses and benefits of mobile devices and apps for healthcare professionals, while Silva et al. in (7) has discussed the importance of using mobile applications to improve health. M-health is becoming an indispensable healthcare tool as borne out by the fact that many ministries of health around the world are providing M-health applications through their official websites.

Alzheimer's disease is one of the health conditions being targeted by mobile application developers. Indeed, the efficacy of using these apps in fighting Alzheimer's has been 
demonstrated by Yasini and Marchand in (8) in a study that followed 15 older adults for 6 months; the results proved that a mobile application could improve cognitive function in elderly patients.

While aging is generally accompanied by functional and cognitive decline, which has an adverse effect on the independence and quality of life of older people, nowadays the most commonly recognized sign of aging is Alzheimer's disease. Alzheimer's is defined as a progressive disease in which the symptoms of dementia gradually worsen over a number of years. In its early stages, memory loss is mild, but with late-stage Alzheimer's individuals lose the ability to carry on a conversation and to respond to their environment (9). According to Alsaqer and Chatterjee (10), the UN has stated that the number of elderly people over the age of 60 will reach 2 billion by 2050 . Moreover, a report by the World Health Organization (WHO, 2015) states that life expectancy is increasing globally and could rise by $0.5 \%$ each year. Hence it is highly likely that Alzheimer's will become much more prevalent. According to the Right Diagnosis website (RightDiagnosis.com), which is one of the world's leading providers of online medical health information, the number of Alzheimer's patients is increasing every year around the world. According to (11), Alzheimer's prevalence in men and women over the age of 85 years is $24 \%$ and $33 \%$, respectively. Therefore, Alzheimer's disease is considered a critical risk around the globe.

In light of the above, and due to the increasing the popularity of mobile applications, both academicians and industry professionals are developing mobile applications that can deal with the various problems associated with Alzheimer's from both the patient's and the caregiver's perspectives. In fact, recently, an enormous number of mobile applications that provide multi-services for Alzheimer's patients and their caregivers have been proposed or have appeared on the market.

In this paper, these mobile applications are analyzed in order to identify the weaknesses and strengths of each application and thereby highlight the research gaps and the consequent opportunities to improve these tools. Finding ways to improve the applications that deal with Alzheimer's disease that will then enhance the overall quality of life of Alzheimer's patients and their families is the main motivation for this research. It should be noted that, in this paper, we focus on mobile applications developed specifically for the Android operating system because, according to the (12) there are three Android users for every iOS user and thus the impact of this research stream numerically speaking may be far greater.

The remainder of this paper is organized as follows: section 2 presents the methodology followed to identify the relevant works for this survey. In section 3 , the collected mobile applications are presented. In section 4 , the results of an analysis of the types of mobile applications are discussed and the gaps in knowledge and potential avenues for further research are identified. Finally, in section 5, a conclusion about the overall direction of this field of research is provided.

\section{Methods}

To ease the search process for our survey, the mobile health applications were classified into two groups: non-academic applications, which were collected by searching Google Play, and academic applications, which were collected by searching Google Scholar. We adopted the following selection criteria to identify the relevant papers for both groups:

Inclusion criteria:

* The title should contain the word "mobile" and any of these words: Alzheimer's, dementia, cognitive. This criterion was adopted because the title should reflect the main contribution of the work;

* The work should deal with the difficulties or at least one of symptoms or problems of Alzheimer's. In other words, the work should provide a real solution and practical service for Alzheimer's patients and/or their caregivers.

Exclusion criteria:

* Works that used sensors to monitor heart rate, body temperature, ECG, respiration rate, tilt and fall, as in (13) were excluded because the aim was to explore simple applications that can be used by everyone.

* Works that provided a contribution that had been discussed and presented clearly in other works were excluded to avoid the redundancy problem.

As noted above, our survey of mobile applications that deal with Alzheimer's disease is limited to Android applications. We believe that this will not restrict the usefulness of our findings in respect of non-academic mobile applications because mobile application companies tend first to release a product on the Android platform and then issue versions for other mobile operating systems. Therefore, by focusing only on Android applications we are not omitting any important application, service, or product that could have been developed for another mobile operating 


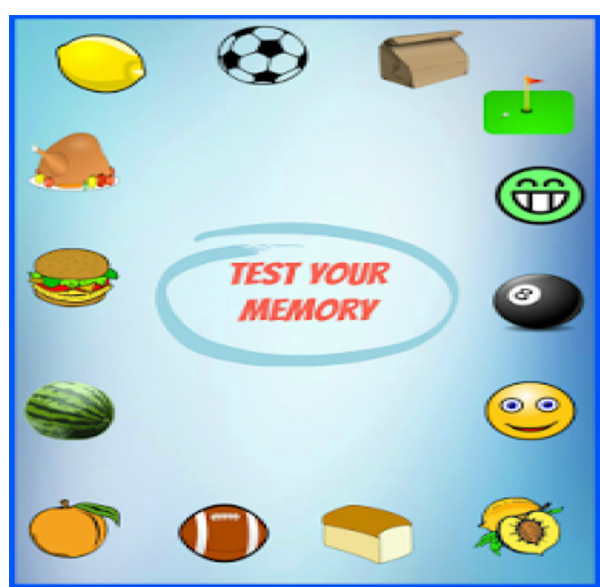

Figure 1 Snapshot of Test Memory Game (16).

system. We chose Google Play as the engine for this part of the search process because it is the primary resource for Android applications. We used Google Play statistics about the number of installs, content rating, and reviewer comments to choose the most popular applications. The use of these three types of statistic resulted in the selection of four applications.

A different methodology was followed to find academic works related to mobile applications for Alzheimer's. We used the Google Scholar search engine because we believe that works that are not cited in Google Scholar are either not academic or are of relatively low quality. The search string used to search the website was: "Alzheimer's + mobile application". The search was limited chronologically to the most recent 10 years, i.e., from 2007 to 2017 in order to capture the most recent works in this growing field of interest. This resulted in the identification of 920 papers. All the papers were deeply investigated, but only a few of them are featured in this paper. The four commercially available apps and a select number of academic works are discussed in the next section.

\section{Types of mobile applications for Alzheimer's}

In this section, the mobile applications that were selected as a result of our survey are presented and discussed. To reflect the above methodology, the section is divided in two parts: non-academic applications and academic applications.

\section{Non-academic applications}

In this section, we present the data that resulted from searching Google Play using the search string "Alzheimer
+ mobile application". We found tens of applications but, as mentioned above, we selected the four most-downloaded applications based on Google Play statistics.

The first application is Backup Memory (14) which is an Android application that works via Bluetooth technology. It allows initially raises the images to the application itself, to upload images of people who are known to the patient, and as soon as such a person approaches the patient, it shows an alert with a number of images related to that person. This helps the patient to remember who the person is more quickly. In addition, Backup Memory uses a global positioning system (GPS) to identify the patient's location.

The second application is Tweri (15), which is an Android application designed in the collaboration with a Spanish association for relatives of people with Alzheimer's disease. Tweri provides a tracking facility for Alzheimer's patients. Tweri (15) use GPS technology to allow caregiver to monitor the patient all the time. The third application is the Test Memory Game (16), which was developed by Xalgo, a software development company. The application aims at helping those with Alzheimer's to improve their memory. It does so through offering exercises that contain shapes, numbers, and letters. The Test Memory Game has five levels and players can move up to the next level after successfully completing the current level. In this game, the player cannot initiate an account, which means he/she needs to start from the beginning every time. Figure 1 shows a snapshot of the Test Memory Game (16).

Finally, the fourth application is called "Prevent Alzheimer with maze" and was developed by Chiquitillo Games (17). The game is intended to help users improve and train their memory through games. There are many games, some for them for enhancing memorizing and some for improving analytical thinking.

Table 1 summarizes the features and technologies of the above applications. By features we mean the functions that are provided by the application and by technologies we mean any technical component that has been added to the Android operating system.

\section{Academic applications}

In this section, we present the data derived from our search of Google Scholar by applying the search string "Alzheimer + mobile application". The key identified works are discussed in order of publication starting with the earliest.

Nearly a decade ago, Bravo et al. (18) developed a near field communication (NFC) application for supporting the caregivers of persons with Alzheimer's. NFC is a short- 
Table 1 Summary of non-academic applications

\begin{tabular}{llll}
\hline No. & Application & Features & Added technology \\
\hline 1 & Backup Memory & $\begin{array}{l}\text { Helps patients to remember quickly by showing them related pictures; } \\
\text { defines patient location }\end{array}$ & Bluetooth; GPS \\
2 & Tweri & Tracks the location of Alzheimer's patients & GPS \\
3 & Test Memory Game & Improves memory by exercises & Just Android application \\
4 & Prevent Alzheimer with maze & Improves and trains memory through games & Just Android application \\
\hline
\end{tabular}

GPS, global positioning system.

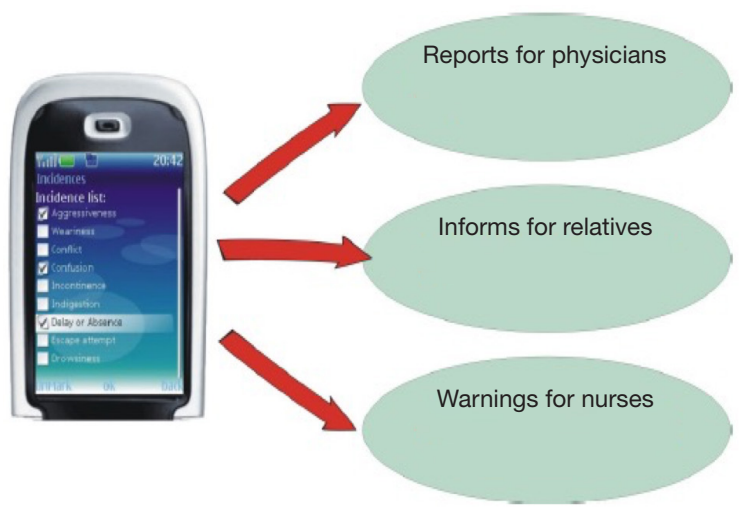

Figure 2 Snapshot of Bravo et al. (18) application.

range radio wave technology that uses a frequency of 13.56 MHz. The application proposed in (18) also utilizes radio frequency identification (RFID) technology. It was developed to restrict and monitor the movements of Alzheimer's patients. In addition, it provides communication tools so that Alzheimer's patients and their caregivers (such as doctors, relatives, and nurses) can interact easily. Figure 2 shows a snapshot of the Bravo et al. (18) application.

In a similar vein, Geddes and Warwick (19) developed a GPS-based safety monitoring application for Alzheimer's patients. The aim of using GPS for Alzheimer's patients is to give caregivers and families a service that can, via a short messaging service (SMS) text message, notify them should their loved one leave their home. The application enables caregivers to set the boundaries within which they think it is safe for the patient to move.

On the other hand, Pavlakis et al. (20) developed a multimedia mobile application that uses sensors to detect whether the user of the mobile device has fallen. After concluding that a fall has occurred, the application informs the patient and starts a counter from 30 to 0 ; at zero, if the patient does not turn off the application because perhaps he/she are too injured or us unconscious, the application sends an SMS to the caregiver or relative containing the geographical location of the patient as well as directions on how to get there on a map. This helps relatives to identify the point of falling and assess how dangerous it may be. Furthermore, the application also allows the patient to directly send an SMS to his/her caregiver containing information about his/her location, if they are able. Figure 3 shows a snapshot of the Pavlakis et al. (20) application.

Hoey et al. (21) developed mobile application to deal with another problem that occurs in patient with Alzheimer's; the wandering problem. The application provides different options based on the situation, such as showing information on the position of the patient or calling the caregiver. Figure 4 shows a snapshot screen of the Hoey et al. (21) application.

Habash et al. (22) proposed an Android-based application to assist doctors in treating Alzheimer's patients. This application provides a communication channel between doctors and their Alzheimer's patients through SMSs and a chat page. In addition, the application includes a medication schedule. Figure 5 shows a snapshot of the Habash et al. (22) application.

Coppol et al. (23) developed a mobile application to help patients with dementia and with Alzheimer's. Their application provides a test for improving cognitive skills through identifying a variety of well-known pictures from nature, such as animals, and of the patient's family, such as sons and daughters. In addition, it provides international samples of music. Figure 6 shows a snapshot of the Coppol et al. (23) application.

On the other hand, Yuce and Gulkesen (24) developed a mobile application for securing and locating an Alzheimer's patient who is outdoors and in a wandering state. The application uses a tracking device installed as a subscriber identity module (SIM) for a global system for mobile 


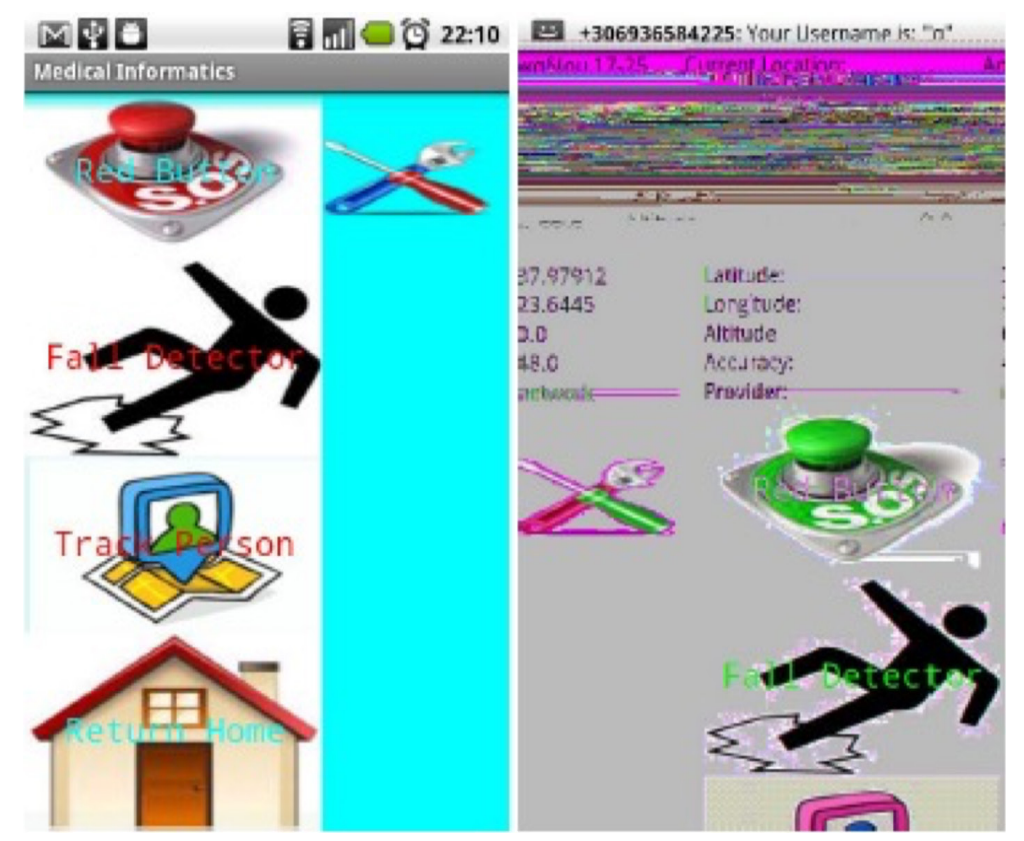

Figure 3 Snapshots of Pavlakis et al. (20) application.
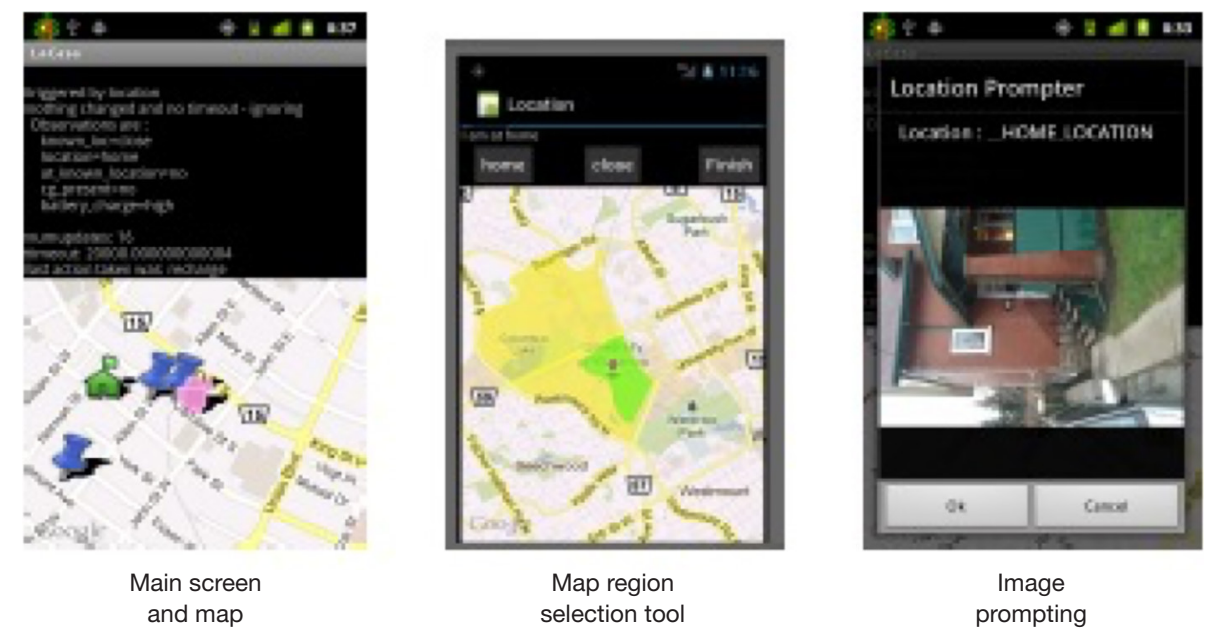

Figure 4 Snapshots of Hoey et al. (21) application.

(GSM) communications network. Along similar lines, Xiao et al. (25) developed a mobile system to remotely monitor the traveling status of the elderly with dementia. The application captures the traveling status by using a built-in smartphone camera with an adhesive fish-eye lens, compass and GPS. Garcia (26) also developed a mobile application for monitoring the elderly and Alzheimer's patients using GPS. The application provides a web-based system through which caregivers can monitor the patient and configure how they want the alarms to be triggered. The web application contains maps in order to define secure zones, sends instant messages to the mobile application, and sets events in a calendar.

Taking a different direction, Griol and Molina (27) developed an Android multimodal application based on natural language processing (NLP) for enhancing the cognitive skills of Alzheimer's patients. The application helps Alzheimer's patients to enhance their memory and 

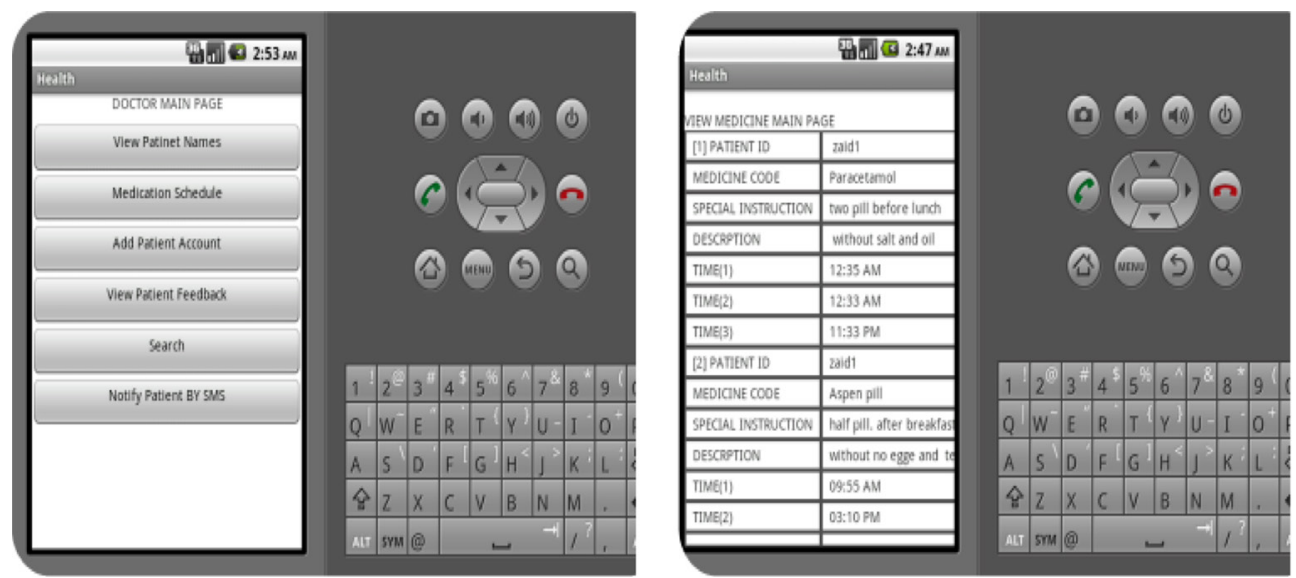

Figure 5 Snapshot of Habash et al. (22) application.

\section{Gallery APP}

Enjoy these beautiful photos from our gallery of images and don't forget to come back for more... Hear the sound of your favorite song while you go trough the photos to experience even bigger

pleasure.

few photo albums:
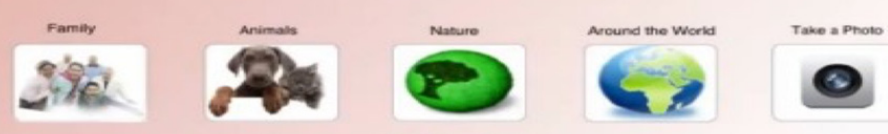

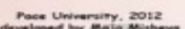

Figure 6 Snapshot of Coppol et al. (23) application.

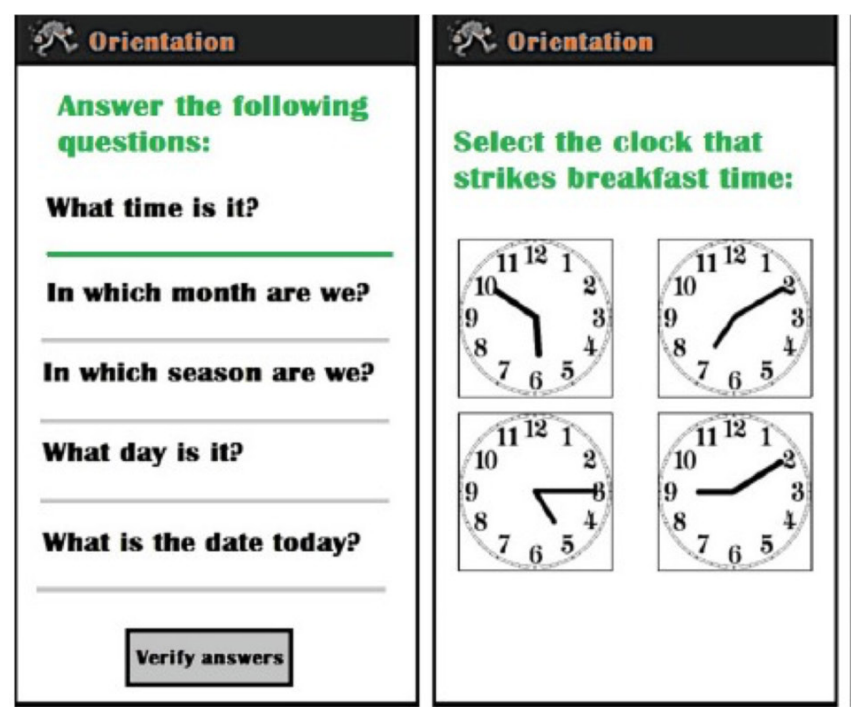

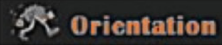

Select the clock that strikes breakfast time:

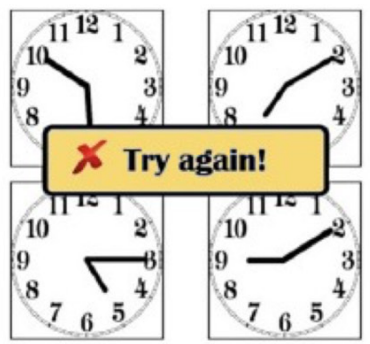

Figure 7 Snapshot of Griol and Molina (27) application. 

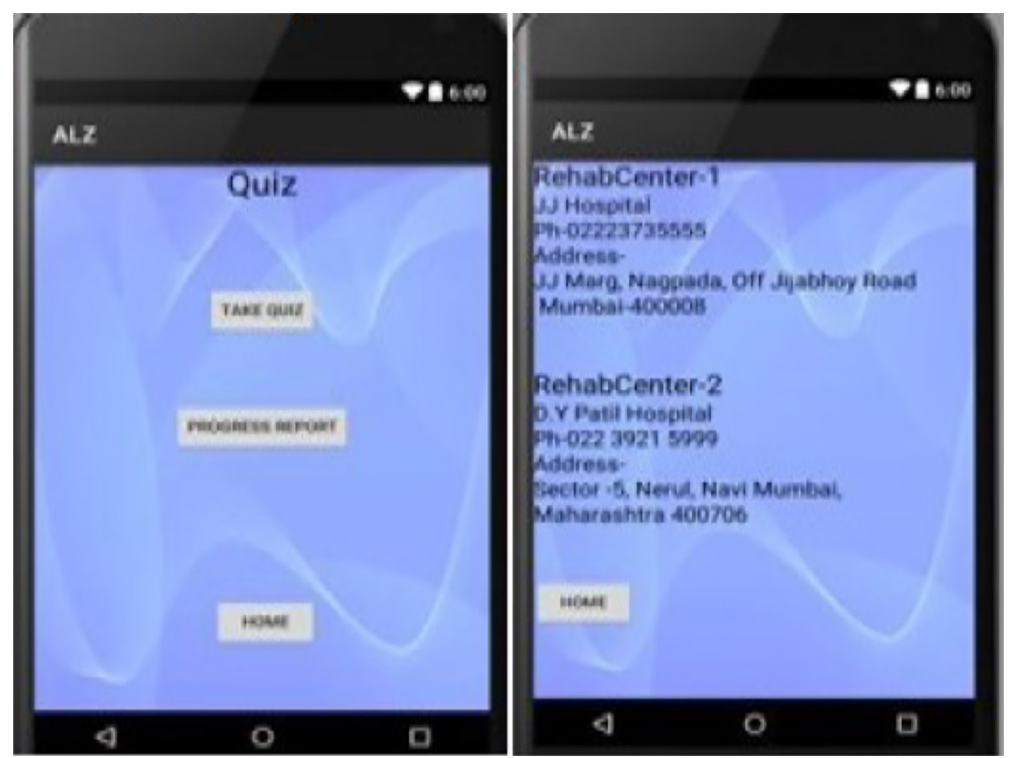

Figure 8 Snapshot of Pirani et al. (11) application.

to stimulate their cognitive abilities by reading a text and answering multiple questions. Figure 7 shows a snapshot of the Griol and Molina (27) application.

In another research stream, Sindi et al. (28) developed an early risk alarm application that calculates the risk factors for dementia and Alzheimer's disease. The system can predict up to 20 years ahead by using data on age, educational level, hypertension, hypercholesterolemia, obesity, and physical inactivity. Moreover, the application provides guidance for risk modification and suggests consulting a healthcare practitioner if needed. It also allows practitioners to discuss preventive measures and monitor risk reduction.

On the other hand, Choon (29) developed an Android application for helping people with Alzheimer's in their daily life, which provides an audio reminder that can help them to do their everyday activities on time. Moreover, this application allows caregivers to have remote access to the Alzheimer's patient's smartphone on which the app is installed to check the history of the performed activities. Pirani et al. (11) also developed an Android-based assistive application for Alzheimer's patients. In this case, the application provides a facility to track the movements of patients through GPS. It also gives medicine and food timing notifications. In addition, it has a daily routine tracker and quiz to increase the cognitive functioning of people with Alzheimer's. Figure 8 shows a snapshot of the application developed by Pirani et al. (11).
Other notable works include that of Méndez-Sanz et al. (30), who developed and evaluated a web-mobile application for calculating the risk of Alzheimer's based on the completion of a short questionnaire, and that of Twidle (31), who developed a text-based mobile game for increasing awareness of Alzheimer's disease. The aim of this application was to improve knowledge of the symptoms and behaviors relating to Alzheimer's disease.

Meanwhile, Duque et al. (32) developed a mobile application for detecting Alzheimer's patients' movement patterns through data provided by accelerometers. The goal of this application was to explore the relationship between these patterns and the stage of Alzheimer's disease. The application was developed based on neural network classifiers and was applied in a case study involving 35 patients. The proposed application achieved a success rate of up to $83 \%$.

On the other hand, Alsaqer and Chatterjee (10) developed mobile application called AdBo to guide elderly patients though appropriate exercises, to measure their cognitive ability before and after the exercise regime, and to track their cognitive improvement over time. This application was created because it is often the case that older people may forget to do exercises or not remember how to do them because of a decline in memory function. Figure 9 shows the AdBo home screen that was developed by Alsaqer and Chatterjee (10).

In other works, Yasini and Marchand (8) evaluated 
the adoption of an application dedicated to cognitive stimulation in the elderly. The application has different games to deal with different cognitive functions such as memory, attention, and concentration. Meanwhile, Quesada-Arencibia et al. (33) developed an application that could act as a channel of communication between doctors and patients in the treatment of mental disorders. As it

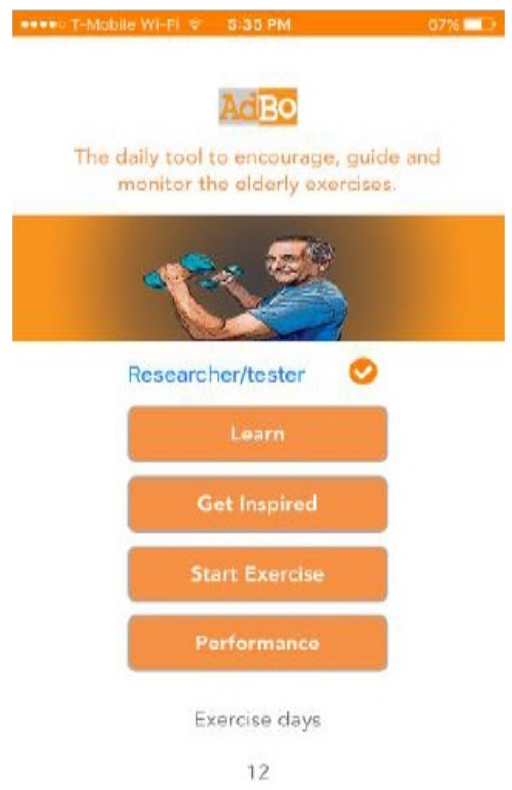

Figure 9 Snapshot of AdBo home screen developed (10). allows doctors to monitor data in real time and have a realtime dialog they can evaluate the mental status of patients more accurately.

More recently, Boyd et al. (34) developed a mobile application for cognitive assessment based on color selection, named it EnCare. This paper has examined the development and usability of EnCare diagnostics for healthy older adults, cognitively impaired and physically impaired individuals. EnCare diagnostics reveals the early stage of Alzheimer's disease, which allows the timely deployment of appropriate treatment. Also, Burns et al. (35) developed a mobile application to assist clinicians in the management of the behavioral and psychological symptoms of dementia through the provision of medical documentation. This application allows doctors to access the medical records of their patients anytime from anywhere. Figure 10 shows a snapshot of the Burns et al. (35) application.

The above-mentioned academic applications are summarized in Table 2, which highlights the features and technology that each uses.

\section{Results}

The above section identified the main features/functions that current M-health applications offer for Alzheimer's patients and the technologies used to deliver them. In this section, we present the results of taking our analysis further.

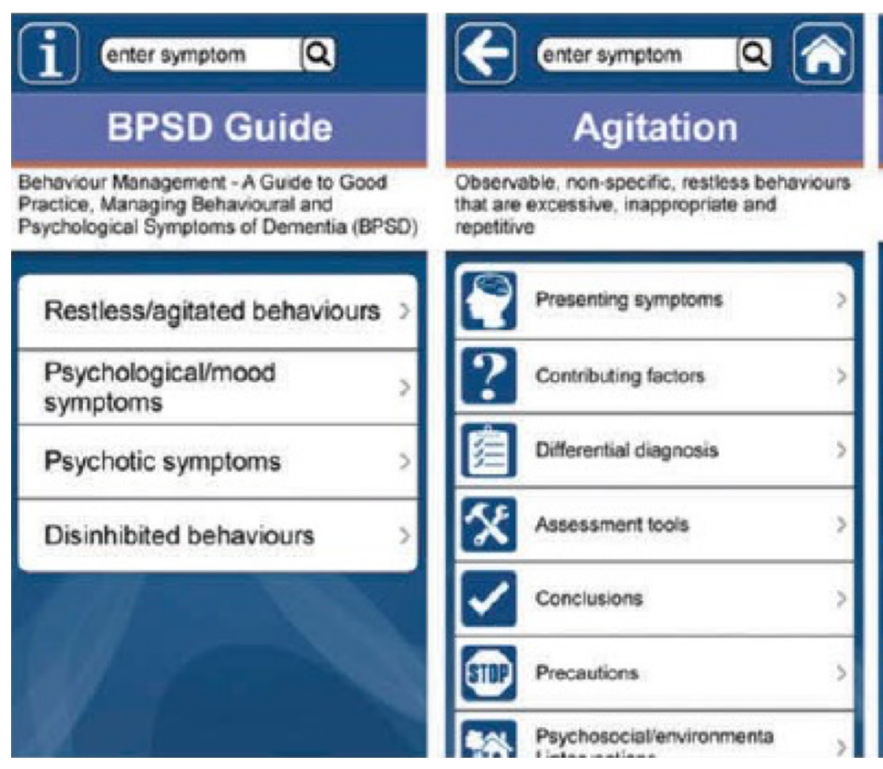

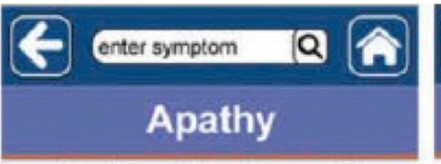

Psychosocial/environmental interventions

Continuous activity programming model of

care

Scientife quality of research Hedect Outcomes. Postive, 1 study

Cued exercise to music

Sowntic quality of resesech L mited Outoomes: Postue: 1 small study

Dancing slor singing (eouples)

Soverstic aualty of research. Limited Qutoomes: Postre: 1 small study

Emotion-onented care based on validation. reminiscence therapy \& sensory stmulation Sowmetic quality of rotearch. Sheng Oucomes: No benofic 2 lage studes

Exercise weh 8 wenout mytrmic music

Scientifo quality of rosearch Limitod Outobmes Equinocat 1 small thoty

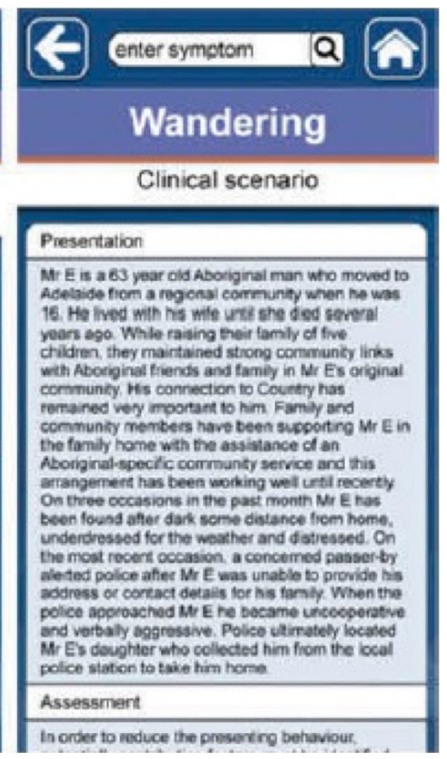

Figure 10 Snapshot of Burns et al. (35) application. 
Table 2 Summary of academic applications

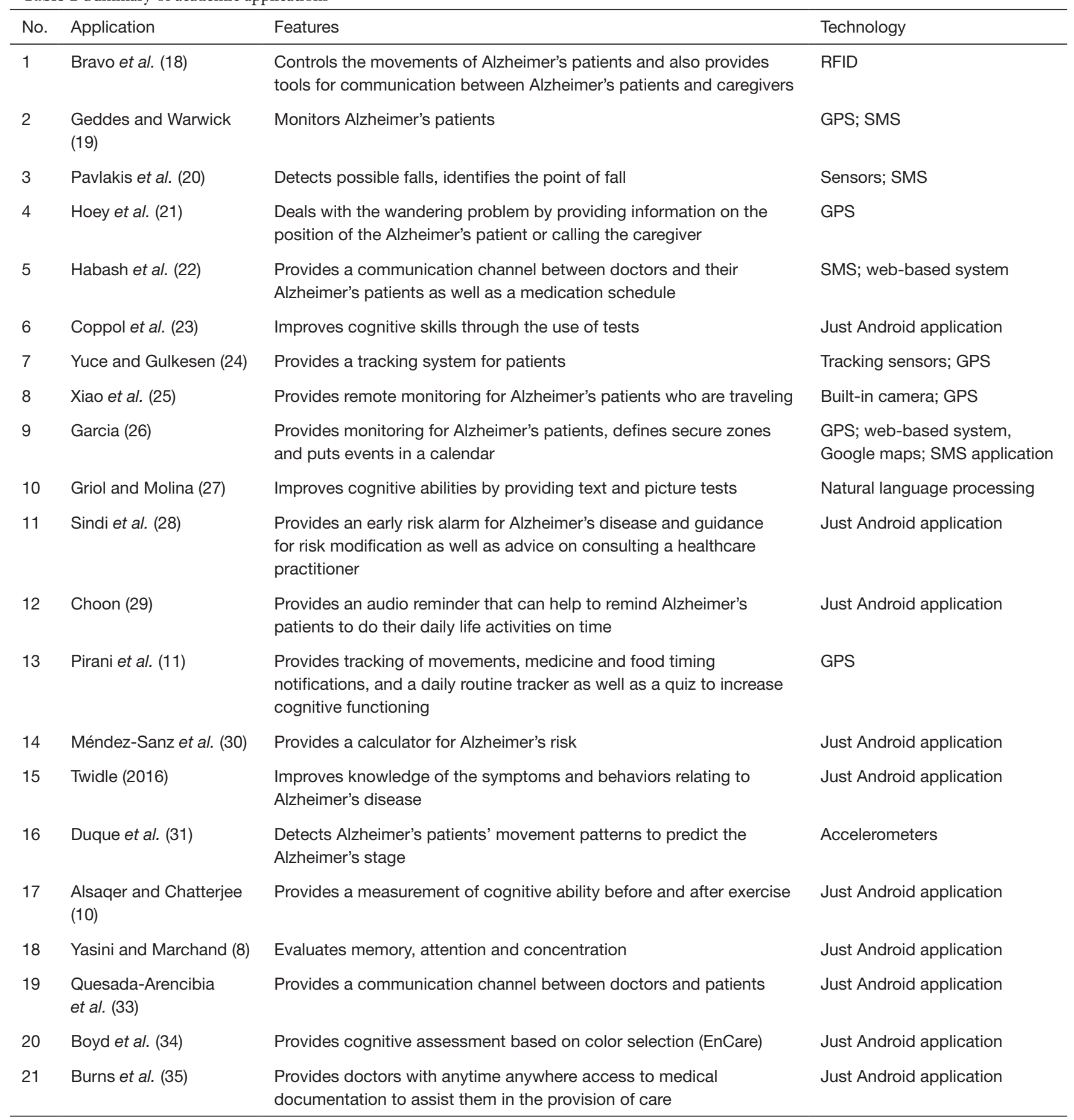

First, we categorized the functions available in the above works in terms of the beneficiary, by which we mean Alzheimer's patient and/or caregiver. The results of this step are shown in Table 3.
Second, we identified four distinct categories into which the services offered by the above M-health applications could be grouped in terms of the services they provide to fight Alzheimer's: (I) enhancing human memory, 
Table 3 M-health functions and beneficiary

\begin{tabular}{|c|c|c|}
\hline No. & Function & Beneficiary \\
\hline 2 & Provides tools for communication between Alzheimer's patients and caregivers & Caregiver/patient \\
\hline 3 & Detects possible falls & Patient \\
\hline 4 & Monitors Alzheimer's patients & Caregiver \\
\hline 6 & Provides guidance for risk modification & Caregiver \\
\hline 7 & Suggests consulting a healthcare practitioner & Caregiver \\
\hline 8 & Defines secure zones for Alzheimer's patients & Caregiver \\
\hline 9 & Puts events in a calendar & Caregiver/patient \\
\hline 12 & Provides an audio reminder that can help Alzheimer's patients to do their daily life activities on time & Patient \\
\hline 13 & Improves knowledge of the symptoms and behaviors relating to Alzheimer's disease & Caregiver/patient \\
\hline 14 & Predicts the Alzheimer's stage & Caregiver/patient \\
\hline 15 & Helps Alzheimer's patients to remember by presenting pictures and videos & Patient \\
\hline 16 & Improves cognitive skills & Patient \\
\hline 17 & Improves and trains memory through games & Patient \\
\hline
\end{tabular}

Table 4 M-health applications for Alzheimer's: categories and associated services

\begin{tabular}{ll}
\hline Category & Associated services \\
\hline $\begin{array}{l}\text { Enhancing human } \\
\text { memory }\end{array}$ & Tests cognitive skills; improves cognitive skills; improves and trains memory through games \\
Ensuring safety & $\begin{array}{l}\text { Controls the movements of Alzheimer's patients; detects possible falls; monitors Alzheimer's patients; defines } \\
\text { secure zones for Alzheimer's patients }\end{array}$ \\
Improving awareness & $\begin{array}{l}\text { Provides early risk alarm for Alzheimer's disease; provides guidance for risk modification; suggests consulting a } \\
\text { healthcare practitioner; predicts the Alzheimer's stage; improves knowledge of symptoms and behaviors relating } \\
\text { to Alzheimer's disease }\end{array}$ \\
Facilitating patients' & $\begin{array}{l}\text { Provides communication tools between Alzheimer's patients and caregivers; puts events in a calendar; provides } \\
\text { daily activities }\end{array}$ \\
\hline
\end{tabular}

(II) ensuring safety, (III) improving awareness, and (IV) facilitating patients' daily activities. Table 4 provides the results.

Then we analyzed the surveyed works in depth and, by also referring to available modern technologies, we identified some further avenues that researchers and practitioners could take in order to provide more useful M-health applications for Alzheimer's patients.
In the following, the research gap and future directions are discussed based on the above four Alzheimer's M-health application categories:

(I) Enhancing human memory: the current applications (in this category) are based on puzzles and games that were developed based on psychological models (36). To move forward, we would suggest three future research directions: 
* Create a measurement tool to define the suitability of the patient for the game before he/she starts playing it. Currently, any patient can play any game without restriction or even guidance, which has an impact on the usefulness of the game;

* Focus more on virtual reality. For instance, virtual reality could provide a virtual environment that relates to the earlier life of the patient, which he/she still remembers well;

* Provide multi-level games in which moving up a level is based on a real measured improvement, and vice versa;

(II) Ensuring safety: the current applications (in this category) use sensors, GPS, or Google Maps. To develop applications for this purpose, we would suggest the following two research directions:

* Provide a direct link to emergency services such as police, ambulance. Adding this feature may result in improving patient safety because it will save time if the patient gets into difficulties, because it cuts out the need to contact the caregiver first and then the caregiver having to contact the service needed;

* Provide identification technology. For instance, provide an identification signal that could help people nearby to recognize the patient; by using Bluetooth technology the patient's smartphone could send a signal to identify him/her to all the people around him/her;

(III) Improving awareness: the current applications (in this category) are based on providing information for the patient or/and his caregiver. For this service category we would suggest three potential research directions:

* Apply Google accelerated mobile pages (AMP) technology. Applying AMP technology will enhance the navigation process, which would allow the patient/caregiver to navigate a huge amount of information in a reasonable time;

* Use summarizing news technology. This technology provides only a summary of the news from different predefined sources, which would make it easier for the patient/caregiver to keep up to date with the latest news;

* Integrate M-health applications with social media. The impact of social media on our daily life is well known. Hence providing
Alzheimer's patients with the chance to interact with others via social media is very important. The M-health applications could provide safe and comfortable channels for patients to communicate with others on social media;

(IV) Facilitating patients' daily activities: the current applications (in this category) are aimed at helping Alzheimer's patients to remember. This is the main problem faced by such patients, hence these applications try to provide different ways to help them. For this key aspect, we would suggest the following two directions for research:

* Integrate M-health applications with intelligent home technologies. The Internet of Things and the new Google operating system for new smart devices (Brillo) offer good potential for M-health application developers to produce full home integration services for Alzheimer's patients;

- Integrate M-health applications with robot technologies. Current developments in robot technologies such as raspberry technology offer good potential for M-health applications. Similarly, an integration of M-health and smart watch technologies could produce useful applications for Alzheimer's patients.

\section{Conclusions}

In this paper, numerous M-health applications that attempt to assist both patients and caregivers in their fight against Alzheimer's were surveyed in order to highlight and explore current and future directions in this field. To aid our data collection process, we classified these mobile applications into two classes-academic and non-academic-and employed a set of selection criteria.

From our analysis of the surveyed papers, we identified the main functions and services that are provided by current $M$-health applications to combat the many facets of Alzheimer's. The papers were analyzed to identify how the applications work and the technology used. Based on this analysis, we were able to present the research foci of current M-health applications. We also categorized the functions provided by these applications based on the intended beneficiary (patient/caregiver). We then grouped these applications into four service categories in order to identify potential future research directions.

The overall aim of this paper was to assist practitioners 
and academicians who are interested in developing M-health applications for Alzheimer's disease by exploring the functions of current applications and suggesting some new directions for research activities. We hope we have achieved this goal and that this paper will be of some benefit to both patients and caregivers.

\section{Acknowledgements}

Authors gratefully acknowledges the support and assistance of the Robotics and Artificial Intelligence lab, Faculty of Computers and Information Technology, University of Tabuk, Saudi Arabia.

\section{Footnote}

Conflicts of Interest: The authors have no conflicts of interest to declare.

\section{References}

1. Liang X, Wang Q, Yang X, et al. Effect of mobile phone intervention for diabetes on glycaemic control: a metaanalysis. Diabet Med 2011;28:455-63.

2. Varshney U. Mobile health: Four emerging themes of research. Decis Support Syst 2014;66:20-35.

3. Baig MM, GholamHosseini H, Connolly MJ. Mobile healthcare applications: system design review, critical issues and challenges. Australas Phys Eng Sci Med 2015;38:23-38.

4. Alotaibi MM, Istepanian R, Philip N. A mobile diabetes management and educational system for type-2 diabetics in Saudi Arabia (SAED). Mhealth 2016;2:33.

5. Luxton DD, McCann RA, Bush NE, et al. mHealth for Mental Health: Integrating Smartphone Technology in Behavioral Healthcare. Prof Psychol Res Pr 2011;42:505-12.

6. Ventola CL. Mobile devices and apps for health care professionals: uses and benefits. P T 2014;39:356-64.

7. Silva BM, Rodrigues JJ, de la Torre Díez I, et al. Mobilehealth: A review of current state in 2015. J Biomed Inform 2015;56:265-72.

8. Yasini M, Marchand G. Adoption and Use of a Mobile Health Application in Older Adults for Cognitive Stimulation. Stud Health Technol Inform 2016;221:13-7.

9. Alzheimer's Association. Alzheimer's Association is a notfor-profit organization. 2016.

10. Alsaqer M, Chatterjee S. AdBo: A mobile application to boost adherence of physical exercises for elderly suffering from cognitive decline. In: Parsons J, Tuunanen T, Venable JR, et al. Editors. Breakthroughs and Emerging Insights from Ongoing Design Science Projects: Researchin-progress papers and poster presentations from the 11th International Conference on Design Science Research in Information Systems and Technology (DESRIST). Canada: St. John, 2016.

11. Pirani Z, Bulakiwala F, Kagalwala M, et al. Android Based Assistive Toolkit For Alzheimer. Procedia Comput Sci 2016;79:143-51.

12. Global web index. Available online: https://www. globalwebindex.net/

13. Megalingam RK, Pocklassery G, Jayakrishnan V, et al. Smartphone based continuous monitoring system for home-bound elders and patients. Available online: https:// ieeexplore.ieee.org/document/6950039/

14. Samsung newsroom. Samsung Volunteers in Tunisia Develop App for Alzheimer's Patients. Available online: https://news.samsung.com/global/samsung-volunteers-intunisia-develop-app-for-alzheimers-patients

15. Tweri. Autonomy for people with Alzheimer's disease and peace of mind for their relatives and caregivers. 2016. Available online: http://www.tweri.com/home.aspx

16. Test memory Game. Developed by Xalgo. 2016. Available online: https://appgrooves.com/android/com. TestMemoryGame/test-memory-game/xalgo/

17. Prevent Alzheimer with maze. Available online: http:// chiquitillo-games.android.informer.com/ko/

18. Bravo J, López-de-Ipiña D, Fuentes C, et al. Enabling NFC technology for supporting chronic diseases: a proposal for alzheimer caregivers. In: Aarts E, Crowley JL, de Ruyter B, et al. Editors. Ambient Intelligence. Berlin, Heidelberg: Springer, 2008;109-25.

19. Geddes J, Warwick K. Cloud based global positioning system as a safety monitor for dementia patients. 2010 IEEE 9th International Conference on Cyberntic Intelligent Systems, Reading, 2010;1-6.

20. Pavlakis P, Alepis E, Virvou M. Intelligent mobile multimedia application for the support of the elderly. 2012 Eighth International Conference on Intelligent Information Hiding and Multimedia Signal Processing, Piraeus, 2012;297-300.

21. Hoey J, Yang X, Quintana E, et al. LaCasa: Location And Context-Aware Safety Assistant. 2012 6th International Conference on Pervasive Computing Technologies for Healthcare (PervasiveHealth) and Workshops, San Diego, CA, 2012;171-4. 
22. Habash ZA, Wan Ishak WH, Omar MH. Android-Based Application to Assist Doctor With Alzheimer's Patient. Proceedings of the 4th International Conference on Computing and Informatics, ICOCI 2013 28-30 August, 2013 Sarawak, Malaysia.

23. Coppol JF, Kowtko MA, Yamagata C, et al. Applying Mobile Application Development to Help Dementia and Alzheimer Patients. Proceedings of Student-Faculty Research Day, CSIS, Pace University, 2013.

24. Yuce YK, Gulkesen KH. CaregiverNet: A Novel Social Support Intervention for Locating and Securing Wandering Alzheimer's Patients As Soon As Possible. 9th International Wireless Communications and Mobile Computing Conference (IWCMC). Cagliari, Sardinia, 2013; Italy.

25. Xiao B, Asghar MZ, Jamsa T, et al. "Canderoid": A mobile system to remotely monitor travelling status of the elderly with dementia. International Joint Conference on Awareness Science and Technology and Ubi-Media Computing (iCAST-UMEDIA), 2013.

26. Garcia JM. Teleassistance outdoor system for the elderly based on Android devices. IEEE International Symposium on Signal Processing and Information Technology(ISSPIT), Athens, 2013; Greece.

27. Griol D, Molina JM. Ambient Assisted Living Mobile Application for Helping People with Alzheimer. Proceeding of International Conference on Practical Applications of Agents and Multi-Agent Systems, 2015; Salamanca, Spain.

28. Sindi S, Calov E, Fokkens J, et al. The CAIDE Dementia Risk Score App: The development of an evidencebased mobile application to predict the risk of dementia. Alzheimers Dement (Amst) 2015;1:328-33.

doi: $10.21037 /$ mhealth.2018.07.03

Cite this article as: Elfaki AO, Alotaibi M. The role of M-health applications in the fight against Alzheimer's: current and future directions. mHealth 2018;4:32.
29. Choon LS. Helper system for managing alzheimer's people using mobile application. Available online: http:// umpir.ump.edu.my/id/eprint/13060/1/FSKKP\%20-\%20 LIM\%20SAY\%20CHOON\%20-\%20CD\%209775.pdf

30. Méndez-Sanz P, Torre-Díez I, López-Coronado M. What is Your Risk of Contracting Alzheimer's Disease? A Telematics Tool Helps you to Predict it. J Med Syst 2016;40:3.

31. Cook B, Twidle P. Increasing Awareness of Alzheimer's Disease through a Mobile Game. 2016 International Conference on Interactive Technologies and Games (ITAG), Notthingham, 2016;55-60.

32. Duque R, Nieto-Reyes A, Martínez C, et al. Detecting human movement patterns through data provided by accelerometers. A case study regarding Alzheimer's disease. In: de Tirajana SB, Canaria G. Editors. Ubiquitous Computing and Ambient Intelligence. Spain: The series Lecture Notes in Computer Science, 2016;56-66.

33. Quesada-Arencibia A, Pérez-Brito E, García-Rodríguez $\mathrm{CR}$, et al. An eHealth information technology platform to help the treatment of mental disorders. Health Informatics J 2016. [Epub ahead of print].

34. Boyd A, Synnott J, Nugent C, et al. Communitybased trials of mobile solutions for the detection and management of cognitive decline. Healthc Technol Lett 2017;4:93-6.

35. Burns K, Jayasinha R, Brodaty H. Evaluation of an Electronic App Developed to Assist Clinicians in the Management of Behavioral and Psychological Symptoms of Dementia (BPSD). Int J Hum Comput Interact 2017;33:902-10.

36. Baddeley AD. Human Memory: Theory and Practice. Boston: Allyn \& Bacon, 1990. 